\title{
Scalable Belief Updating for Urban Air Quality Modeling and Prediction
}

\author{
XIUMING LIU, Uppsala University, Uppsala, Sweden \\ EDITH NGAI, The University of Hong Kong, Pokfulam Road, Hong Kong, China \\ DAVE ZACHARIAH, Uppsala University, Uppsala, Sweden
}

\begin{abstract}
Air pollution is one of the major concerns in global urbanization. Data science can help to understand the dynamics of air pollution and build reliable statistical models to forecast air pollution levels. To achieve these goals, one needs to learn the statistical models which can capture the dynamics from the historical data and predict air pollution in the future. Furthermore, the large size and heterogeneity of today's big urban data pose significant challenges on the scalability and flexibility of the statistical models. In this work, we present a scalable belief updating framework that is able to produce reliable predictions, using over millions of historical hourly air pollutant and meteorology records. We also present a non-parametric approach to learn the statistical model which reveals interesting periodical dynamics and correlations of the dataset. Based on the scalable belief update framework and the non-parametric model learning approach, we propose an iterative update algorithm to accelerate Gaussian process, which is notorious for its prohibitive computation with large input data. Finally, we demonstrate how to integrate information from heterogeneous data by regarding the beliefs produced by other models as the informative prior. Numerical examples and experimental results are presented to validate the proposed method.
\end{abstract}

CCS Concepts: • Mathematics of computing $\rightarrow$ Bayesian computation; Nonparametric statistics; Stochastic processes;

Additional Key Words and Phrases: Urban air quality, statistical model, scalability, heterogeneous data, belief updating, gaussian process

ACM Reference format:

Xiuming Liu, Edith Ngai, and Dave Zachariah. 2020. Scalable Belief Updating for Urban Air Quality Modeling and Prediction. ACM/IMS Trans. Data Sci. 2, 1, Article 2 (December 2020), 19 pages.

https://doi.org/10.1145/3402903

\section{INTRODUCTION}

Air pollution in urban environment has been a well-known problem for a very long history globally. Ever since the Victorian Britain, we have read Charles Dickens' records about the urban air

This research work was supported by The Swedish Research Council through projects under Contracts 621-2016-06079, 2017-04543, and 2018-05040.

Authors' addresses: X. Liu and D. Zachariah, Department of Information Technology, Uppsala University, Box 337, SE-751 05 Uppsala, Sweden; emails: \{xiuming.liu, dave.zachariah\}@it.uu.se; E. Ngai (corresponding author), Room 608, Chow Yei Ching Building, Department of Electrical and Electronic Engineering, The University of Hong Kong, Pokfulam Road, Hong Kong, China; email: chngai@eee.hku.hk.

Permission to make digital or hard copies of all or part of this work for personal or classroom use is granted without fee provided that copies are not made or distributed for profit or commercial advantage and that copies bear this notice and the full citation on the first page. Copyrights for components of this work owned by others than the author(s) must be honored. Abstracting with credit is permitted. To copy otherwise, or republish, to post on servers or to redistribute to lists, requires prior specific permission and/or a fee. Request permissions from permissions@acm.org.

(c) 2020 Copyright held by the owner/author(s). Publication rights licensed to ACM.

2577-3224/2020/12-ART2 \$15.00

https://doi.org/10.1145/3402903 
pollution in London as a side effect of the industrial revolution. In today's China, the number of motor vehicles reaches nearly 300 million, ${ }^{1}$ which echoes the increasing concerns about the air quality in major cities around the globe. The World Health Organization (WHO) reports that the air pollution raises a high risk of various health problems to citizens and generate a significant amount of economic cost to the society [21]. Data science can contribute tools and knowledge to help solving the air pollution problem. For example, predicting the future air quality based on statistical models and historical data is very useful for both city administrators and citizens.

However, it is non-trivial to provide reliable predictive results for urban air quality, due to that a large number of random factors (various human activities and meteorology conditions) can produce significant impacts on the urban air qualities. To tackle this challenge, the following research questions need to be addressed. First, how do we construct an interpretable statistical model which is capable of revealing interesting dynamic patterns and predict future dynamics? Second, how do we utilize large and heterogeneous historical data for producing the predictions? To answer those questions, in this work, we focus on two aspects of the data analysis for urban air quality: descriptive statistical modeling of the large historical measurements and scalable prediction methods for future air quality levels based on big data.

The increasing amount of connected sensors and intelligent devices provide us access to large and heterogeneous data. Very often, the data include the historical measurements of concentrations of air pollutants and other relevant records such as meteorological records (e.g., temperature, wind speed, etc.). In some cities, efforts of measuring air quality have been carried out for decades, which provides us a large dataset (over millions of hourly records). As we will show through this article, historical data contains rich information about the dynamics of air pollution levels in the urban area, and can be used to produce accurate predictions of future air quality. In this work, we take the hourly concentrations of nitrogen-dioxides $\left(\mathrm{NO}_{2}\right)$ and particulates with a diameter between 2.5 and 10 micrometers $\left(\mathrm{PM}_{10}\right)$ at several monitoring stations to be a measure of air quality $[1,22]$.

To extract useful information from those large and heterogeneous data, we use the descriptive and predictive statistical models. A descriptive model reveals interesting patterns in the historical measurements, while a predictive model produces predictions for future air quality based on the inputs. In general, a statistical model connect measurements with the unobserved variables, using probability distributions. Recalling the Box's dictum "all models are wrong but some are useful" [6], we are looking for models that are capable of accommodating different types of data in large amounts and produce accurate predictions of future air quality levels.

Among many candidates for statistical modeling of time-series data (such as the concentrations of air pollutants indexed by time), Gaussian process (GP) has been widely applied for its simplicity and flexibility [12,17]. A GP is a model of stochastic processes under the assumption of an arbitrary set of samples drawn from the process is jointly Gaussian distributed [4, 25]. Applying GP for regression analysis consists of two steps: model learning and prediction. The model learning step refers to the procedure of building GP models based on the training data. A GP is uniquely specified by its mean and covariance (or kernel) functions. To obtain the GP model, typically one has to accomplish three tasks: first, study and discover the dynamics in the training data; second, design the mean and covariance functions with hyper-parameters which encode the discovered dynamics; finally determine values for hyper-parameters via various estimation methods, such as maximum likelihood estimation (MLE). However, this parametric approach of learning GP can be ineffective in big data applications due to the difficulty of optimizing values of parameters in the likelihood function which contains very large covariance matrices. Both model learning and prediction steps necessitate computing inverses of the $N \times N$ covariance matrix, which require a storage

\footnotetext{
${ }^{1}$ Statistics from the Traffic Management Bureau of the Public Security Ministry, PRC, January 2017.
} 
and runtime that scale as $O\left(N^{2}\right)$ and $O\left(N^{3}\right)$, respectively. This fact makes it computationally infeasible to apply the full GP regression to the urban air quality scenario with big data.

In this work, we will show how the GP model can be learned from big data in an empirical and non-parametric fashion which is simple and effective. We build on the belief updating formulation in Bissiri et al. [5] which generalizes the standard Bayesian approach. We develop a scalable formulation that overcomes the computational limits of the standard approach for predicting urban air quality. The resulting method is capable of producing accurate and reliable predictions based on a large historical data records. The contribution of this work is summarized as follows:

- We present a fully non-parametric method for modeling the urban air quality and meteorological data. This model reveals interesting dynamics of the urban air quality.

- We propose a scalable belief update method to produce predictions based on very large and heterogeneous datasets, using the composite likelihood and informative priors.

- We propose a recursive belief update algorithm which can mitigate the computational complexity bottleneck of classic Gaussian process model. We also show that the popular sparse GP models are special cases of the scalable belief updating method, when using different alternatives of the standard likelihood function.

This article is organized as follows: In Section 2, we review the related work of statistical modeling for time-series data, including Gaussian process and a more general belief updating framework. In Section 3, we present descriptive modeling and analysis for the urban air pollution and meteorological data. Based on the revealed patterns in the statistical models, we propose a scalable belief updating method for air pollution prediction in Section 4. Numerical examples, experimental results, and discussions are provided in Section 5. Finally, we conclude this article and discuss the future work.

\section{Notations}

$\boldsymbol{x}_{\boldsymbol{t}}$ is a column vector indexed by the vector of time stamps, $\boldsymbol{x}_{\boldsymbol{t}}=\left[\begin{array}{llll}x_{1} & x_{2} & \ldots & x_{N}\end{array}\right]^{\top} . \boldsymbol{\mu}_{\boldsymbol{t}}$ is the column vector of means, $\boldsymbol{\mu}_{\boldsymbol{t}}=\left[\mu_{1} \mu_{2} \ldots \mu_{N}\right]^{\top}$, and $\Sigma_{\boldsymbol{t}, \boldsymbol{t}}$ is the covariance matrix of the vector $\boldsymbol{x}_{\boldsymbol{t}}$ with the $(i, j)$ entry $\mathbb{E}\left[\left(x_{i}-\mu_{i}\right)\left(x_{j}-\mu_{j}\right)\right]$. Similarly, $\Sigma_{t_{1}, t_{2}}$ is the cross-covariance between vectors $\boldsymbol{x}_{\boldsymbol{t}_{1}}$ and $\boldsymbol{x}_{\boldsymbol{t}_{2}}$.

When the data $x_{t}$ is divided into $K$ segments and the length of a segment is $M$, we denoted the divided data as $x_{t}=\left\{\boldsymbol{x}_{\boldsymbol{t}_{1}}, \ldots, \boldsymbol{x}_{\boldsymbol{t}_{K}}\right\}$, where $\boldsymbol{x}_{\boldsymbol{t}_{k}}$ is the $k$ th segment. For example, if $\boldsymbol{x}_{\boldsymbol{t}}$ represents the total 40 weeks of hourly data and is divided into $K=4$ segments, then $\left\{\boldsymbol{x}_{\boldsymbol{t}_{1}}, \boldsymbol{x}_{\boldsymbol{t}_{2}}, \boldsymbol{x}_{\boldsymbol{t}_{3}}, \boldsymbol{x}_{\boldsymbol{t}_{4}}\right\}$ represents the first, the second, the third, and the fourth 10 weeks of hourly data, respectively. And each segment of data will contain $10 \times 7 \times 24$ hourly measurements.

$D_{\mathrm{KL}}(q \| p)$ is the KL divergence between two probability distributions $q$ and $p$.

\section{RELATED WORK}

A large number of studies have been performed on analyzing big urban air quality data. In this section, we review previous studies of urban air data modeling, Gaussian process (GP) regression, and the belief updating framework.

Urban Air Quality Monitoring and Modeling. There are many research work on monitoring the urban air quality with sensors and forecasting the future air quality based on machine learning models. Zheng et al. [32] present the general concept of urban computing with applications in air quality monitoring and prediction. In [33] and [34], the authors present an air quality prediction model using many urban-related features such as the point-of-interest, the traffic data, and the 
mobility trajectories. In [27], Shaban et al. present an air-quality monitoring system with various machine learning prediction models. Jain et al. [15] apply the graph signal processing to analyze the environmental impact of burning different types of heating oils in big cities.

With the recent advance of deep learning methods, many works apply various deep learning models for forecasting urban air quality. Among those models, the recurrent neural networks (RNN) [9] and its variant, the long short-term memory (LSTM), are the most popular type of models for time series prediction [2, 8, 13, 18, 23, 31]. Yi et al. [31] proposed a distributed deep fusion network, which takes spatial transformed air quality index and meteorology data as input and generates predicted future air quality index. Qi et al. [23] proposed a deep air learning method which consists of an auto-encoder with feature selection and a semi-supervised spatial-temporal regression network. In [18], Liang et al. presented a multi-layer attention network for spatial-temporal sensor data regression.

Gaussian Process Regression. Among a large numbers of candidates for modeling time-series data, Gaussian process (GP) is a widely applied model for regression analysis and prediction [4, 25]. A GP model is specified by its mean and covariance functions, instead of a finite set of parameters [4]. A GP model defines a prior distribution for the concentrations of pollutants $\boldsymbol{x}_{*}$ with time inputs $\boldsymbol{t}^{*}$. Given a set of historical concentrations $\boldsymbol{x}_{\boldsymbol{t}}$ with time inputs $\boldsymbol{t}$, the posterior distribution for unobserved $\boldsymbol{x}_{*}$ is a conditional Gaussian distributions with closed-form solutions for mean values and covariances. By designing the mean and covariance functions, GP allows us to represent various properties learned from the air pollution data.

Nevertheless, given a set of observations $x_{t}$ with size $N$, the inversion of covariance matrices (size of $N \times N)$ in GP regression requires a runtime that scales as $O\left(N^{3}\right)$ [24]. This fact limits the application of GP regressions to small or moderated data sets. A great effort of developing scalable GP for big data has been made in the last decade [19, 24]. Sparse GP (SGP) regression is based the idea of using relatively small set of observations to represent the information in the entire set of observations [24]. Two examples of SGP are the subset of data (SoD) method and the subset of regression (SoR) method. The SoD is perhaps the most intuitive method. In general, we select $M$ samples from $N$ observations $(M \ll N)$, such that the running time of matrix inversion is reduced to $O\left(M^{3}\right)$ [24]. The selection scheme of a subset of observations has a clearly major impact on the accuracy of inferences. Instead of randomly selecting observations, one might choose the most informative samples by maximizing the information gain [17, 26]. The disadvantage of the SoD methods is that only a small part of the training data will be used. In order to include more information from the available observations, the SoR methods, such as the "fully independent conditional (FIC)", are proposed [24, 29]. The SoR methods use the inducing variables to encode more information from the observations for inferences. However, in order to avoid the inversion of large covariance matrices, approximations are required to model the conditional distribution for the entire observations sets given inducing variables. This approximation can be expensive in the sense of information loss.

Belief Distribution Update. Bissiri et al. [5] proposed a general belief updating framework, which minimizes the expected loss plus a regularization term. If the loss function is defined as the negative $\log$-likelihood function given the past observations $\boldsymbol{x}_{\boldsymbol{t}}$, then the result equals the standard posterior belief distribution. This formulation highlights a major bottleneck of the standard Bayesian approach (such as the GP model): the evaluation of the full joint likelihood with a large dataset. Furthermore, the generalization of standard Bayesian methods allows us to design the prior which contains information from heterogeneous and non-structural data, for instance, the impact of meteorological condition on the urban air quality. 


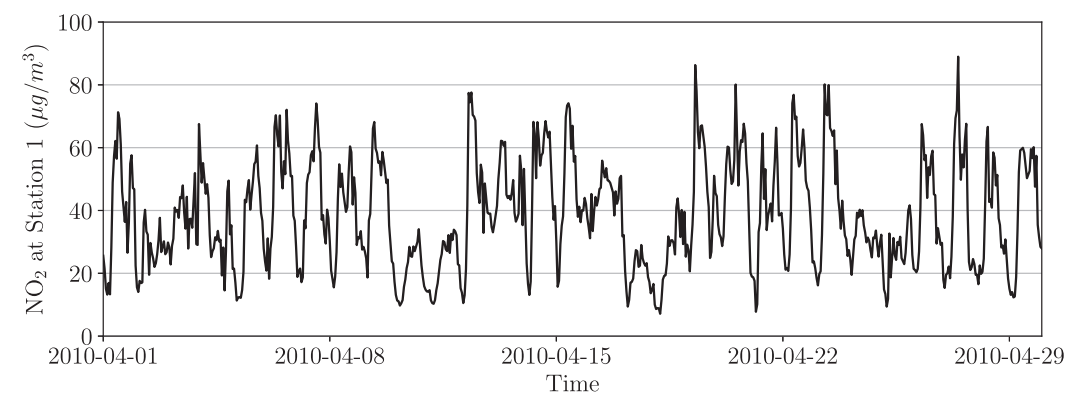

Fig. 1. An example of $\mathrm{NO}_{2}$ hourly measurements collected from Station 1 during April 2010.

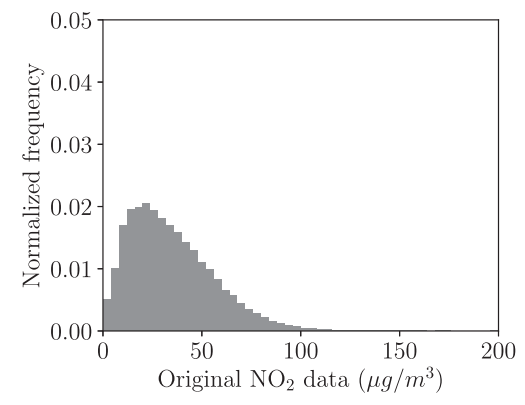

(a) Before transform

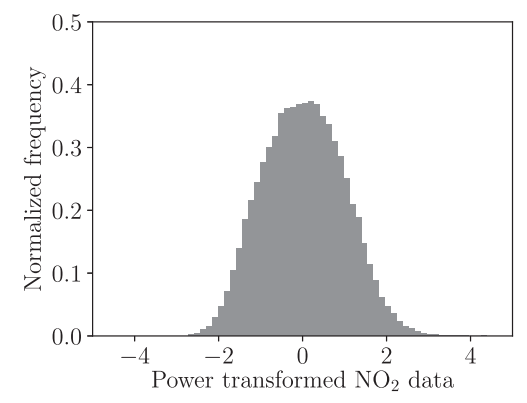

(b) After transform

Fig. 2. Power transformation of the $\mathrm{NO}_{2}$ measurements.

\section{DESCRIPTIVE STATISTIC OF THE AIR POLLUTION DATA}

In this section, we explore various statistical properties of the urban air quality data: the lognormality of air pollution concentrations; the periodic patterns; and the impact of meteorology conditions.

The dataset ${ }^{2}$ studied in this work contains two types of air pollutants, $\mathrm{NO}_{2}$ and $\mathrm{PM}_{10}$, which are measured by the hourly averages $\left(\mu \mathrm{g} / \mathrm{m}^{3}\right)$ from five different stations in the city. The total time period of the measurements last 10 years, from January 1, 2006 to December 31, 2015. An example of the air pollutants data are shown in Figure 1. The dataset also contains five types meteorology data (hourly average) collected at a region station during the same time period, namely radiation $\left(\mathrm{W} / \mathrm{m}^{2}\right)$, relative humidity $(\%)$, temperature $\left({ }^{\circ} \mathrm{C}\right)$, wind speed $(\mathrm{m} / \mathrm{s})$, and wind direction (azimuth degree $^{\circ}$ ). The other important side information of the dataset is the time vector itself. For example, by looking up the calendar, one can easily determine the weekday of a given time point. The dataset thus has a considerably large size (nearly a million data points) and contains heterogeneous data types.

\subsection{Power Transformation of the Original Measurements}

Before proceeding to further analysis, we shall have a glance at the distributions of the $\mathrm{NO}_{2}$ and $\mathrm{PM}_{10}$ data. Since the air pollutants are measured by concentrations, the data are non-negative. Furthermore, the data are highly skewed toward to zero and have long tails into the high concentrations (see Figure 2(a)). This non-Gaussian distribution imposes challenges for modeling and prediction of the data.

\footnotetext{
$\overline{{ }^{2} \text { Available at: http://slb.nu/slbanalys/. }}$
} 


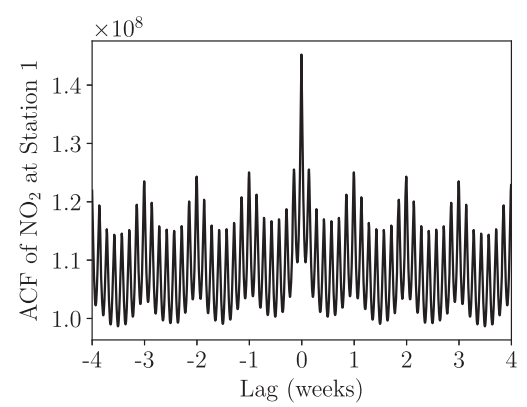

(a) ACF of $\mathrm{NO}_{2}$

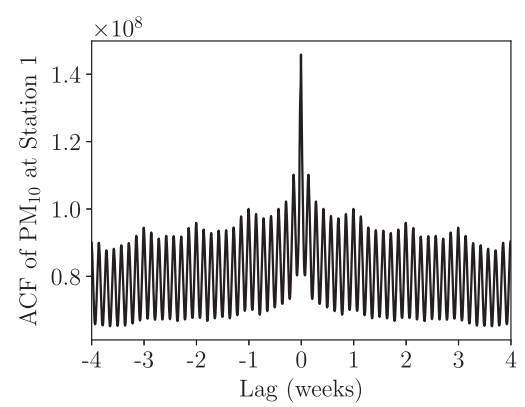

(b) ACF of $\mathrm{PM}_{10}$

Fig. 3. The auto-covariance function (ACF) of the $\mathrm{NO}_{2}$ and $\mathrm{PM}_{10}$ data.

To make the data more Gaussian-like, we apply the power transformation $[7,30]$ to the original measurements. The transformed data are centered around zero (Figure 2(b)), and follow a distribution with the bell curve. Models (for example, the Gaussian process) then can be constructed based on the log-transformed data. Note that the predictive results need to be inversely transformed to be read as proper units of air pollutants concentrations $\left(\mu \mathrm{g} / \mathrm{m}^{3}\right)$.

\subsection{Identify the Periodical Patterns}

Since the human activity (for instance, the road traffic) is the major source of air pollutants in urban areas, the air-quality data shows different dynamics during different hours and weekdays. In the following, we explore those dynamics and present a quantitative analysis of the periodical patterns in the urban air pollution data.

To begin with, we calculate the sample auto-covariance function (ACF) for each pollutant. Following the definition in [28], the sample ACF for a pollutant, $\boldsymbol{x}_{\boldsymbol{t}}$, is given by

$$
\widehat{\operatorname{cov}}(\Delta t)=\frac{1}{N} \sum_{t=1}^{N} \mathbb{E}\left[\left(x_{t+\Delta t}-\mu_{x}\right)\left(x_{t}-\mu_{x}\right)\right],
$$

where $\mu_{x}=\frac{1}{N} \sum_{t=1}^{N} x_{t}$ is the sample mean of $\boldsymbol{x}_{t}$, and $\Delta t$ is the lag. The ACFs for $\mathrm{NO}_{2}$ and $\mathrm{PM}_{10}$ from Station 1 are visualized in Figure 3, which evidently reveals the periodical patterns: for both $\mathrm{NO}_{2}$ and $\mathrm{PM}_{10}$ there are exactly seven peaks within a week, representing the daily patterns; for $\mathrm{NO}_{2}$, the peaks at lag of weeks stands out from peaks of days, indicating that different weekdays have significant impacts on the $\mathrm{NO}_{2}$ level in the city.

The power spectral density (PSD), which is the Fourier transform of the ACF (the WienerKhincin theorem, see [10]), describes how the power of data distributed in the spectral domain. The PSDs of the $\mathrm{NO}_{2}$ and $\mathrm{PM}_{10}$ data are visualized in Figure 4, which reveals a few interesting characteristics: first of all, for both $\mathrm{NO}_{2}$ and $\mathrm{PM}_{10}$, the daily periodicity is the most significant pattern, indicating that the human activity is indeed the major factor for urban air quality; secondly, comparing to $\mathrm{NO}_{2}, \mathrm{PM}_{10}$ shows much weaker weekly pattern, but contains a strong annually periodicity, which can be related to the usage of winter tyres in Nordic countries; finally, the PSD of $\mathrm{NO}_{2}$ even reveals 12- and 8-hour patterns, which are possibly contributed by the rush hours of the urban road traffic.

\subsection{Analysis of the Impact from Meteorological Conditions}

Another important factor of the urban air quality is the meteorological conditions [14]. To investigate the impact of meteorological conditions on the concentrations of air pollutants, we calculate 


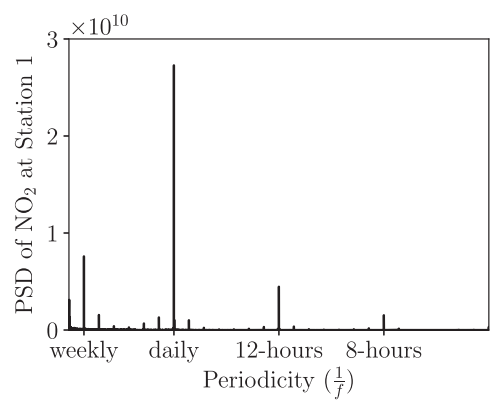

(a) PSD of $\mathrm{NO}_{2}$

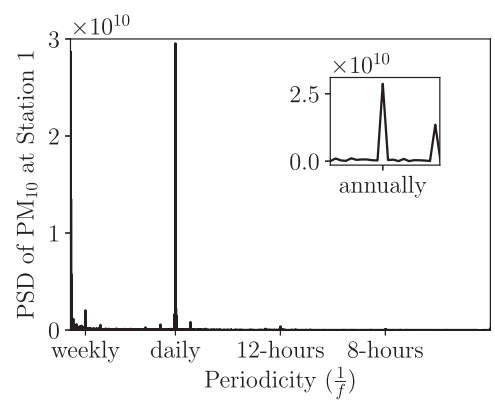

(b) PSD of $\mathrm{PM}_{10}$

Fig. 4. The power spectral density (PSD) of the $\mathrm{NO}_{2}$ and $\mathrm{PM}_{10}$ data.

Table 1. Correlations between the Air Pollutants and the Meteorology Conditions

\begin{tabular}{lcllllll}
\hline \multirow{2}{*}{ Data } & \multicolumn{7}{c}{ Correlation } \\
\cline { 2 - 8 } & 1 & 2 & 3 & 4 & 5 & 6 & 7 \\
\hline 1. $\mathrm{NO}_{2}$ & 1 & & & & & & \\
2. $\mathrm{PM}_{10}$ & 0.344 & 1 & & & & & \\
3. Radiation & -0.091 & 0.178 & 1 & & & & \\
4. $\mathrm{RH}$ & 0.107 & $\mathbf{- 0 . 2 3 8}$ & -0.669 & 1 & & & \\
5. Temperature & $\mathbf{- 0 . 3 0 0}$ & -0.082 & 0.633 & -0.368 & 1 & & \\
6. Wind direction & 0.138 & 0.043 & 0.108 & 0.148 & 0.103 & 1 & \\
7. Wind speed & $\mathbf{- 0 . 4 1 4}$ & -0.051 & 0.014 & -0.099 & 0.056 & 0.045 & 1 \\
\hline
\end{tabular}

the Pearson correlation coefficients ${ }^{3}$ in Table 1. In this calculation, we select data measured at noon of business days, such that the impact of human activities is isolated. According to Table 1, for $\mathrm{NO}_{2}$, the negative correlations with two meteorology data, temperature and wind speed, are significant; for $\mathrm{PM}_{10}$, the negative correlation with relative humidity is notable.

Given the above analysis of correlations, it is important to consider the shift of distributions for air pollutants, when the meteorology conditions change. For example, the joint distribution of $\mathrm{NO}_{2}$ and temperature is visualized in Figure 5(a). When the temperature changes, we observe that the conditional distribution of $\mathrm{NO}_{2}$ shifts: the distribution of $\mathrm{NO}_{2}$ given low temperatures $(\leq 0)$ has a higher average value than the distributions under other conditions (see Figure 5(b)).

Nevertheless, the meteorology conditions for the future are missing: the conditional distribution, $p\left(\boldsymbol{x}_{*} \mid \boldsymbol{y}_{*}\right)$, is inaccessible due to the unknown meteorology data $\boldsymbol{y}_{*}$. There are two possible solutions: first, if the forecast of meteorology conditions, $\widehat{\boldsymbol{y}}_{*}$, is available, one can approximate $p\left(\boldsymbol{x}_{*} \mid \boldsymbol{y}_{*}\right)$ with $p\left(\boldsymbol{x}_{*} \mid \widehat{\boldsymbol{y}}_{*}\right)$; second, given the observed meteorology history $\boldsymbol{y}_{\boldsymbol{t}}$, the conditional distribution $p\left(\boldsymbol{x}_{*} \mid \boldsymbol{y}_{\boldsymbol{t}}\right)$ can also be used as the side information for predicting $\boldsymbol{x}_{*}$. The technical details will be presented in the following section.

\section{SCALABLE BELIEF UPDATING FOR AIR POLLUTION PREDICTION}

In this section, we present the main results of scalable belief updating for predicting urban air quality. We start by formulating the prediction problem with the belief updating framework, then

\footnotetext{
${ }^{3}$ For the wind direction, the circular-linear correlation is calculated, see [20].
} 


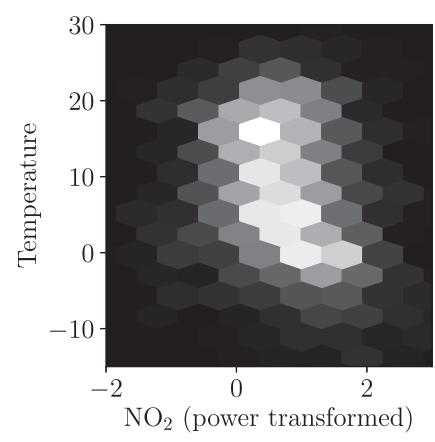

(a) The joint density: $p(x, y)$

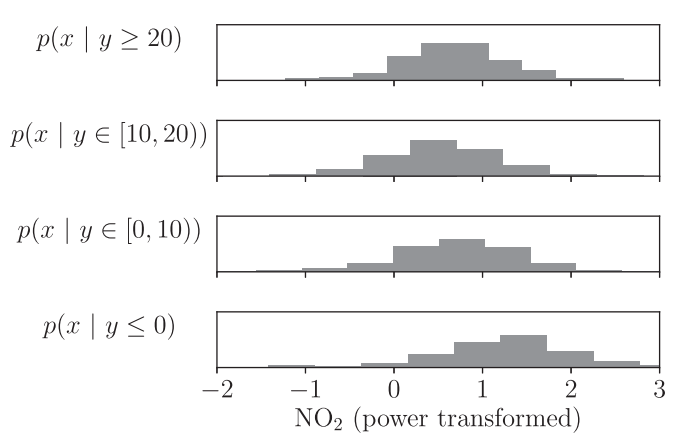

(b) Conditioning on the meteo data: $p(x \mid y)$

Fig. 5. The shift of $\mathrm{NO}_{2}(x)$ distribution due to the change of temperature $(y)$. Here, only the data at noon of business days are selected, such that the comparison is separated from the factor of human activities.

extend to the scalable method for handling big data, and finally present the method for integrating heterogeneous data.

\subsection{Bayesian Prediction as Belief Updating}

Based on the general belief updating framework [5], given the historical observations $\boldsymbol{x}_{\boldsymbol{t}}$, we seek the updated belief distribution

$$
\hat{q}\left(\boldsymbol{x}_{*}\right)=\underset{q\left(\boldsymbol{x}_{*}\right)}{\arg \min } \mathbb{E}\left[\ell\left(\boldsymbol{x}_{*} ; \boldsymbol{x}_{\boldsymbol{t}}\right)\right]+D_{\mathrm{KL}}\left(q\left(\boldsymbol{x}_{*}\right) \| p\left(\boldsymbol{x}_{*}\right)\right),
$$

where

$$
\ell\left(x_{*} ; x_{t}\right)=-\ln p\left(x_{t} \mid x_{*}\right) .
$$

That is, the loss function is defined as the negative log-likelihood of data $x_{t}$. Using this definition, the updated belief distribution is given by

$$
\begin{aligned}
\hat{q}\left(\boldsymbol{x}_{*}\right) & =\underset{q\left(\boldsymbol{x}_{*}\right)}{\arg \min } \int-q\left(\boldsymbol{x}_{*}\right) \ln p\left(\boldsymbol{x}_{\boldsymbol{t}} \mid \boldsymbol{x}_{*}\right) d \boldsymbol{x}_{*}+\int q\left(\boldsymbol{x}_{*}\right) \ln \frac{q\left(\boldsymbol{x}_{*}\right)}{p\left(\boldsymbol{x}_{*}\right)} d \boldsymbol{x}_{*} \\
& =\underset{q\left(\boldsymbol{x}_{*}\right)}{\arg \min } \int q\left(\boldsymbol{x}_{*}\right) \ln \frac{q\left(\boldsymbol{x}_{*}\right)}{p\left(\boldsymbol{x}_{\boldsymbol{t}} \mid \boldsymbol{x}_{*}\right) p\left(\boldsymbol{x}_{*}\right)} d \boldsymbol{x}_{*} \\
& =\underset{q\left(\boldsymbol{x}_{*}\right)}{\arg \min } D_{\mathrm{KL}}\left(q\left(\boldsymbol{x}_{*}\right) \| p\left(\boldsymbol{x}_{\boldsymbol{t}} \mid \boldsymbol{x}_{*}\right) p\left(\boldsymbol{x}_{*}\right)\right) \\
& =\frac{p\left(\boldsymbol{x}_{\boldsymbol{t}} \mid \boldsymbol{x}_{*}\right) p\left(\boldsymbol{x}_{*}\right)}{\int p\left(\boldsymbol{x}_{\boldsymbol{t}} \mid \boldsymbol{x}_{*}\right) p\left(\boldsymbol{x}_{*}\right) d \boldsymbol{x}_{*}},
\end{aligned}
$$

which recovers the standard Bayesian posterior. From this derivation, one can argue that the Bayesian prediction is indeed a special case of the belief updating, when the negative log-likelihood is selected as the loss function, and the prior distribution is used as the regularization term.

GP Prediction. Recall that the transformed air pollution data approximately follows a Gaussian distribution (see Figure 2), the dynamic of air pollution is modeled by the Gaussian process,

$$
x_{t} \sim \mathcal{G P}\left(\mu(t), \operatorname{cov}\left(t, t^{\prime}\right)\right),
$$

where the mean function $\mu(t)$ and the covariance function $\operatorname{cov}\left(t, t^{\prime}\right)$ are estimated using the nonparametric sample mean and covariance (for example, Figure 3). Given this GP model, the posterior 
in Equation (4) can be written in the following closed form,

$$
\begin{aligned}
p\left(\boldsymbol{x}_{*} \mid \boldsymbol{x}_{\boldsymbol{t}}\right) & =\frac{p\left(\boldsymbol{x}_{\boldsymbol{t}} \mid \boldsymbol{x}_{*}\right) p\left(\boldsymbol{x}_{*}\right)}{\int p\left(\boldsymbol{x}_{\boldsymbol{t}} \mid \boldsymbol{x}_{*}\right) p\left(\boldsymbol{x}_{*}\right) d \boldsymbol{x}_{*}} \\
& =\mathcal{N}\left(\boldsymbol{\mu}_{* \mid t}, \Sigma_{* \mid \boldsymbol{t}}\right),
\end{aligned}
$$

where

$$
\begin{gathered}
\boldsymbol{\mu}_{* \mid \boldsymbol{t}}=\boldsymbol{\mu}_{*}+\Sigma_{*, t} \Sigma_{\boldsymbol{t}, \boldsymbol{t}}^{-1}\left(\boldsymbol{x}_{\boldsymbol{t}}-\boldsymbol{\mu}_{\boldsymbol{t}}\right), \\
\Sigma_{* \mid \boldsymbol{t}}=\Sigma_{*}-\Sigma_{*, t} \Sigma_{\boldsymbol{t}, \boldsymbol{t}}^{-1} \Sigma_{\boldsymbol{t}, *} .
\end{gathered}
$$

The predictive mean $\boldsymbol{\mu}_{* \mid t}$ can be used as the point prediction of the future air pollution level, and the predictive variance $\Sigma_{* \mid t}$ serves as a measure of uncertainty for the prediction result.

\subsection{Handling Big Data: The Composite Likelihood}

However, as we discussed previously, the calculation of the standard posterior in Equation (4) can be computationally prohibitive, when the size of observed data is very large. Take the GP posterior as an example, given the observed data $x_{t}$ of size $N$, the inverse of the covariance matrix $\Sigma_{t, t}^{-1}$ takes $O\left(N^{2}\right)$ storage and $O\left(N^{3}\right)$ running time. For a dataset which contains more than a few thousands of data points, the computation becomes infeasible for most numerical software tools and common hardware platforms.

Consider that the large dataset, $\boldsymbol{x}_{\boldsymbol{t}}$, is divided into $K$ segments,

$$
x_{t}=\left\{x_{t_{1}}, \ldots, x_{t_{K}}\right\},
$$

where $x_{t_{k}}$ is the $k$ th segment. The size of each data segment is denoted by $M=\frac{N}{K}$. To extend the framework in Equation (2) for scalable processing of big data, we propose the following method. Let the loss function be the negative composite likelihood,

$$
\ell\left(\boldsymbol{x}_{*} ; \boldsymbol{x}_{\boldsymbol{t}}\right)=-\ln \prod_{k=1}^{K} p\left(\boldsymbol{x}_{\boldsymbol{t}_{k}} \mid \boldsymbol{x}_{*}\right)
$$

Using this loss function, the updated belief distribution is given by

$$
\begin{aligned}
\hat{q}\left(\boldsymbol{x}_{*}\right) & =\underset{q\left(\boldsymbol{x}_{*}\right)}{\arg \min } \int-q\left(\boldsymbol{x}_{*}\right) \ln \prod_{k=1}^{K} p\left(\boldsymbol{x}_{\boldsymbol{t}_{k}} \mid \boldsymbol{x}_{*}\right) d \boldsymbol{x}_{*}+\int q\left(\boldsymbol{x}_{*}\right) \ln \frac{q\left(\boldsymbol{x}_{*}\right)}{p\left(\boldsymbol{x}_{*}\right)} d \boldsymbol{x}_{*} \\
& =\underset{q\left(\boldsymbol{x}_{*}\right)}{\arg \min } \int q\left(\boldsymbol{x}_{*}\right) \ln \frac{q\left(\boldsymbol{x}_{*}\right)}{\prod_{k=1}^{K} p\left(\boldsymbol{x}_{\boldsymbol{t}_{k}} \mid \boldsymbol{x}_{*}\right) p\left(\boldsymbol{x}_{*}\right)} d \boldsymbol{x}_{*} \\
& =\frac{\prod_{k=1}^{K} p\left(\boldsymbol{x}_{\boldsymbol{t}_{k}} \mid \boldsymbol{x}_{*}\right) p\left(\boldsymbol{x}_{*}\right)}{\int \prod_{k=1}^{K} p\left(\boldsymbol{x}_{\boldsymbol{t}_{k}} \mid \boldsymbol{x}_{*}\right) p\left(\boldsymbol{x}_{*}\right) d \boldsymbol{x}_{*}} .
\end{aligned}
$$

The last equality is obtained by recognizing that

$$
\int q\left(\boldsymbol{x}_{*}\right) \ln \frac{q\left(\boldsymbol{x}_{*}\right)}{\prod_{k=1}^{K} p\left(\boldsymbol{x}_{t_{k}} \mid \boldsymbol{x}_{*}\right) p\left(\boldsymbol{x}_{*}\right)} d \boldsymbol{x}_{*}=D_{\mathrm{KL}}\left(q\left(\boldsymbol{x}_{*}\right) \| \prod_{k=1}^{K} p\left(\boldsymbol{x}_{\boldsymbol{t}_{k}} \mid \boldsymbol{x}_{*}\right) p\left(\boldsymbol{x}_{*}\right)\right),
$$

and the KL divergence achieves the minimum (zero) when $q\left(\boldsymbol{x}_{*}\right) \propto \prod_{k=1}^{K} p\left(\boldsymbol{x}_{\boldsymbol{t}_{k}} \mid \boldsymbol{x}_{*}\right) p\left(\boldsymbol{x}_{*}\right)$. Finally, $\int \prod_{k=1}^{K} p\left(\boldsymbol{x}_{t_{k}} \mid \boldsymbol{x}_{*}\right) p\left(\boldsymbol{x}_{*}\right) d \boldsymbol{x}_{*}$ is the normalization factor.

The updated belief $\hat{q}\left(\boldsymbol{x}_{*}\right)$ can be evaluated in a recursive manner. In each step, the updated belief from the previous step is used as the new prior. Combining with the likelihood of a new segment 


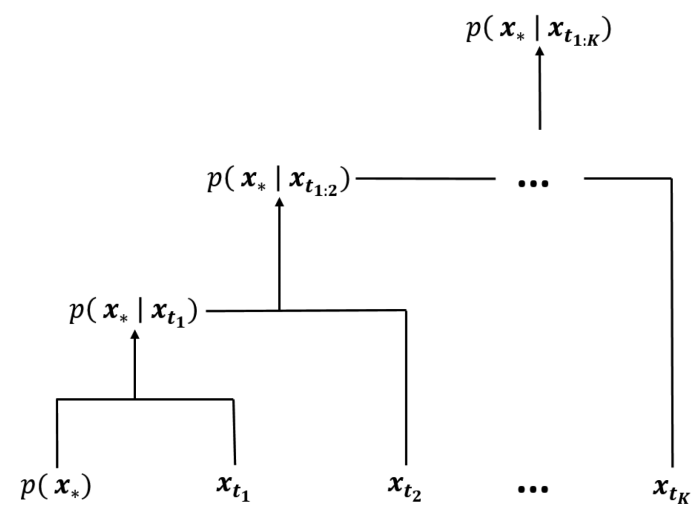

Fig. 6. An illustration of the recursive belief update in Equation (11). The large dataset $x_{t}$ (size $N$ ) is divided into $K$ segments: $x_{t_{1}}, \ldots, x_{t_{K}}$, where each segment of the dataset contains $M=\frac{N}{K}$ data points.

of data, the new posterior is produced. Mathematically, let $p\left(\boldsymbol{x}_{*} \mid \boldsymbol{x}_{t_{1: k-1}}\right)$ be the approximated posterior using data segments $\left\{\boldsymbol{x}_{t_{1}}, \ldots, \boldsymbol{x}_{t_{k-1}}\right\}$. Given the data segment $\boldsymbol{x}_{\boldsymbol{t}_{k}}$, the updated belief is

$$
p\left(\boldsymbol{x}_{*} \mid \boldsymbol{x}_{\boldsymbol{t}_{1: k}}\right)=\frac{p\left(\boldsymbol{x}_{\boldsymbol{t}_{k}} \mid \boldsymbol{x}_{*}\right) p\left(\boldsymbol{x}_{*} \mid \boldsymbol{x}_{\boldsymbol{t}_{1: k-1}}\right)}{\int p\left(\boldsymbol{x}_{\boldsymbol{t}_{k}} \mid \boldsymbol{x}_{*}\right) p\left(\boldsymbol{x}_{*} \mid \boldsymbol{x}_{\boldsymbol{t}_{1: k-1}}\right) d \boldsymbol{x}_{*}} .
$$

An illustration of the recursive update is shown in Figure 6.

Scalable GP Prediction Using the Composite Likelihood. Again, when the data distribution is Gaussian, the above updated belief distribution can be evaluated in closed form. The recursive method for obtaining the updated belief with the GP model is presented in the following. For each $k$ data segment:

(1) The previous posterior $p\left(\boldsymbol{x}_{*} \mid \boldsymbol{x}_{\boldsymbol{t}_{1: k-1}}\right)=\mathcal{N}\left(\boldsymbol{\mu}_{* \mid t_{1: k-1}}, \Sigma_{* \mid \boldsymbol{t}_{1: k-1}}\right)$ is regarded as the new prior. For $k=1$, let $p\left(\boldsymbol{x}_{*}\right)=\mathcal{N}\left(\boldsymbol{\mu}_{*}, \Sigma_{*, *}\right)$ be the initial prior.

(2) Evaluate the likelihood of a single data segment $\boldsymbol{x}_{k}$. The mean and the covariance of this likelihood are specified by this conditional model $p\left(\boldsymbol{x}_{k} \mid \boldsymbol{x}_{*}\right)=\mathcal{N}\left(\boldsymbol{\mu}_{\boldsymbol{t}_{k} \mid *}, \Sigma_{\boldsymbol{t}_{k} \mid *}\right)$, where

$$
\begin{gathered}
\boldsymbol{\mu}_{\boldsymbol{t}_{k} \mid *}=\boldsymbol{\mu}_{\boldsymbol{t}_{k}}+\Sigma_{\boldsymbol{t}_{k}, *} \Sigma_{*, *}^{-1}\left(\boldsymbol{\mu}_{* \mid \boldsymbol{t}_{1: k-1}}-\boldsymbol{\mu}_{*}\right), \\
\Sigma_{\boldsymbol{t}_{k} \mid *}=\Sigma_{\boldsymbol{t}_{k}, \boldsymbol{t}_{k}}-\Sigma_{\boldsymbol{t}_{k}, *} \Sigma_{*, *}^{-1} \Sigma_{*, \boldsymbol{t}_{k}} .
\end{gathered}
$$

Note that since $\boldsymbol{x}_{*}$ is unknown, its previous posterior mean, $\boldsymbol{\mu}_{* \mid \boldsymbol{t}_{1: k-1}}$, is regarded as the latest observation of $\boldsymbol{x}_{*}$.

(3) The new posterior, $p\left(\boldsymbol{x}_{*} \mid \boldsymbol{x}_{t_{1: k}}\right)=\mathcal{N}\left(\boldsymbol{\mu}_{* \mid t_{1: k}}, \Sigma_{* \mid t_{1: k}}\right)$, is obtained via the Bayes rule. The posterior mean and covariance are given by

$$
\begin{gathered}
\boldsymbol{\mu}_{* \mid t_{1: k}=} \boldsymbol{\mu}_{* \mid t_{1: k-1}}+\sum_{* \mid t_{1: k-1}} \boldsymbol{H}_{k}^{\top} \boldsymbol{G}_{k}^{-1}\left[\boldsymbol{x}_{k}-\boldsymbol{\mu}_{k}-\boldsymbol{H}_{k}\left(\boldsymbol{\mu}_{* \mid t_{1: k-1}}-\boldsymbol{\mu}_{*}\right)\right], \\
\sum_{* \mid t_{1: k}}=\sum_{* \mid t_{1: k-1}}-\sum_{* \mid t_{1: k-1}} \boldsymbol{H}_{k}^{\top} \boldsymbol{G}_{k}^{-1} \boldsymbol{H} \Sigma_{* \mid t_{1: k-1}},
\end{gathered}
$$

where

$$
\begin{gathered}
\boldsymbol{H}_{k}=\Sigma_{\boldsymbol{t}_{k}, *} \Sigma_{*, *}^{-1}, \\
\boldsymbol{G}_{k}=\Sigma_{\left.\boldsymbol{t}_{k}\right|^{*}}+\boldsymbol{H}_{k} \Sigma_{* \mid \boldsymbol{t}_{1: k-1}} \boldsymbol{H}_{k}^{\top} .
\end{gathered}
$$




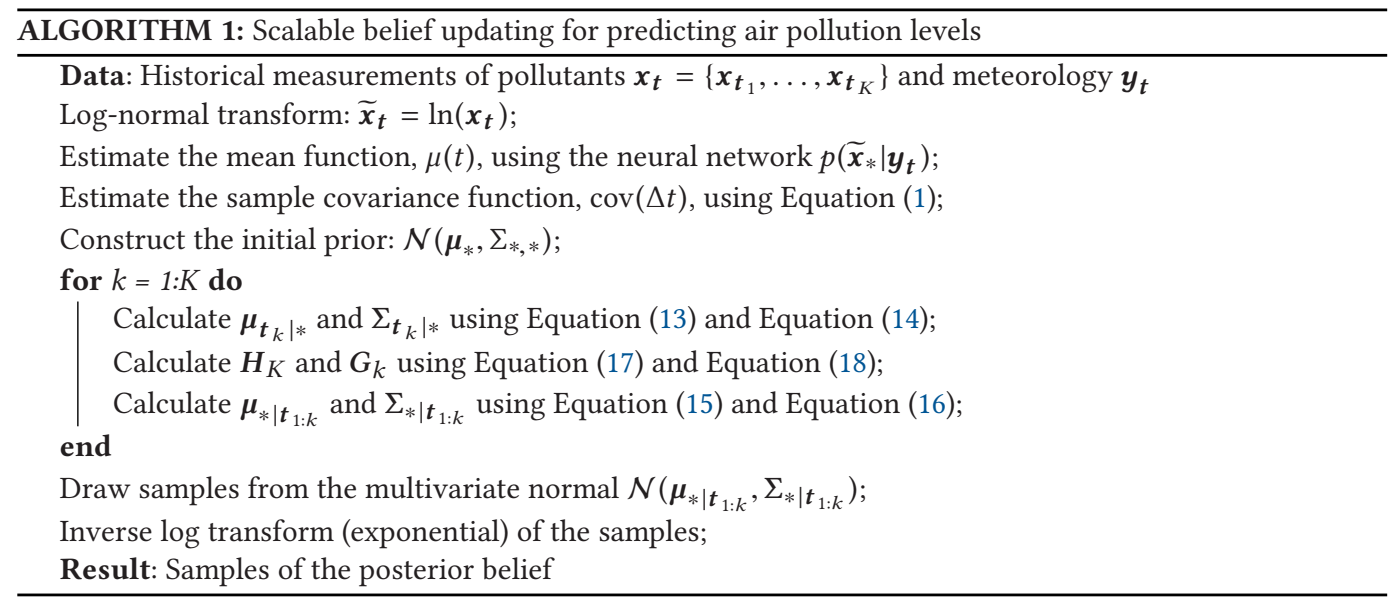

In the above recursive updating procedure, the matrix $G_{k}$ is of size $M$ and therefore the inverse operation has the complexity of $O\left(M^{3}\right)$. Considering that there are in total $K$ data segments, the overall runtime of the above updating procedure scales with $O\left(K \times\left(\frac{N}{K}\right)^{3}\right)$. If the total size of the data $N$ is fixed, then increasing the number of segmentation $K$ will significantly accelerate the updating procedure.

Sparse GP as a Special Case of the Scalable Belief Updating. As we discussed previously, sparsification is another popular scheme to extend standard Bayesian prediction to large datasets. For GP models, the sparse GP (SGP) [24, 29] is a well-known technique. Following the idea of using approximated loss function in Equation (2), we now show that the SGP can be regarded a special case of the scalable belief updating.

The SGP is formulated using a set of latent "inducing variables" $\boldsymbol{u}$ with a joint distribution $p\left(\boldsymbol{x}, \boldsymbol{u} \mid \boldsymbol{x}_{*}\right)$. By assuming that $\boldsymbol{x}$ is independent of $\boldsymbol{x}_{*}$ when given $\boldsymbol{u}$, the joint distribution can be factorized into $p(\boldsymbol{x} \mid \boldsymbol{u}) p\left(\boldsymbol{u} \mid \boldsymbol{x}_{*}\right)$. Then the implicit loss function used in SGP is based on marginalizing out $\boldsymbol{u}$ from the joint distribution:

$$
\ell_{\mathrm{SGP}}\left(\boldsymbol{x} ; \boldsymbol{x}_{*}\right)=-\ln \int_{\mathcal{U}} p(\boldsymbol{x} \mid \boldsymbol{u}) p\left(\boldsymbol{u} \mid \boldsymbol{x}_{*}\right) d \boldsymbol{u},
$$

using a composite likelihood $p(\boldsymbol{x} \mid \boldsymbol{u})=\prod_{b=1}^{B} p\left(\boldsymbol{x}_{b} \mid \boldsymbol{u}\right)$. The SGP posterior is

$$
\begin{aligned}
p_{\mathrm{SGP}}\left(\boldsymbol{x}_{*} \mid \boldsymbol{x}\right) & =\underset{q\left(\boldsymbol{x}_{*}\right)}{\arg \min } \int-q\left(\boldsymbol{x}_{*}\right) \ln \int_{\mathcal{U}} \prod_{b=1}^{B} p\left(\boldsymbol{x}_{b} \mid \boldsymbol{u}\right) p\left(\boldsymbol{u} \mid \boldsymbol{x}_{*}\right) d \boldsymbol{u} d \boldsymbol{x}_{*}+\int q\left(\boldsymbol{x}_{*}\right) \ln \frac{q\left(\boldsymbol{x}_{*}\right)}{p\left(\boldsymbol{x}_{*}\right)} d \boldsymbol{x}_{*} \\
& =\frac{p\left(\boldsymbol{x}_{*}\right) \int_{\mathcal{U}} \prod_{b=1}^{B} p\left(\boldsymbol{x}_{b} \mid \boldsymbol{u}\right) p\left(\boldsymbol{u} \mid \boldsymbol{x}_{*}\right) d \boldsymbol{u}}{\int_{\mathcal{U}} \widetilde{p}(\boldsymbol{x} \mid \boldsymbol{u}) p(\boldsymbol{u}) d \boldsymbol{u}} .
\end{aligned}
$$

This formulation reproduces the fully or partial independent training conditional (FITC or PITC) approximations depending on the size of the segments $\boldsymbol{x}_{b}$, cf. [24, 29]. The inducing variables are targeting a compressed representation of the data and the information is transferred to the belief distribution of $\boldsymbol{x}_{*}$ via $p\left(\boldsymbol{u} \mid \boldsymbol{x}_{*}\right)$. Thu, $\boldsymbol{u}$ must be carefully selected so as to transfer the maximum amount of information about the training data $x$. The optimal placement of inducing variables involves a challenging combinatorial optimization problem and but can be tackled using greedy search heuristics [26]. 


\subsection{Integrate Information from Heterogeneous Data}

As we discussed in the previous section, the meteorology data contains information for the air pollution level. In the following, we integrate those heterogeneous data into the scalable belief updating framework.

Let $\boldsymbol{y}_{\boldsymbol{t}}$ be the observed meteorology data (for example, the meteorological condition from the day prior to the day to be predicted). Due to the missing of the future meteorological condition, we use $\Phi\left(\boldsymbol{x}_{*} \mid \boldsymbol{y}_{t}\right)$ as an approximation of the conditional mean $\mathbb{E}\left[p\left(\boldsymbol{x}_{*} \mid \boldsymbol{y}_{*}\right)\right]$, where $\Phi\left(\boldsymbol{x}_{*} \mid \boldsymbol{y}_{\boldsymbol{t}}\right)$ is a deep neural network. Denote the output of the network as

$$
\widehat{\boldsymbol{\mu}}_{* \mid \boldsymbol{y}_{\boldsymbol{t}}} \triangleq \Phi\left(\boldsymbol{x}_{*} \mid \boldsymbol{y}_{t}\right)
$$

we evaluate the mean squared error and define

$$
\widehat{\Sigma}_{* \mid \boldsymbol{y}_{\boldsymbol{t}}} \triangleq \mathbb{E}\left[\left(\boldsymbol{x}_{*}-\widehat{\boldsymbol{\mu}}_{* \mid \boldsymbol{y}_{\boldsymbol{t}}}\right)^{2}\right] .
$$

And the conditional distribution $p\left(\boldsymbol{x}_{*} \mid \boldsymbol{y}_{*}\right)$ is approximated by

$$
p\left(\boldsymbol{x}_{*} \mid \boldsymbol{y}_{*}\right) \approx p\left(\boldsymbol{x}_{*} \mid \boldsymbol{y}_{t}\right)=\mathcal{N}\left(\widehat{\boldsymbol{\mu}}_{* \mid \boldsymbol{y}_{t}}, \widehat{\Sigma}_{* \mid \boldsymbol{y}_{t}}\right) .
$$

Finally, the obtained conditional distribution $p\left(\boldsymbol{x}_{*} \mid \boldsymbol{y}_{t}\right)$ is used as the prior in the scalable belief updating method:

$$
\hat{q}\left(\boldsymbol{x}_{*} \mid \boldsymbol{y}_{\boldsymbol{t}}\right)=\underset{q\left(\boldsymbol{x}_{*}\right)}{\arg \min } \int-q\left(\boldsymbol{x}_{*}\right) \ln \prod_{k=1}^{K} p\left(\boldsymbol{x}_{\boldsymbol{t}_{k}} \mid \boldsymbol{x}_{*}\right) d \boldsymbol{x}_{*}+D_{\mathrm{KL}}\left(q\left(\boldsymbol{x}_{*}\right) \| p\left(\boldsymbol{x}_{*} \mid \boldsymbol{y}_{\boldsymbol{t}}\right)\right) .
$$

Based on the deterministic input of weekdays, we switch between two NNs: one is trained using data from weekdays and the other is trained using data from weekends and holidays. The inputs of each NN are the previous 24-hours meteorology conditions: radiation $\left(\mathcal{R}^{+}\right)$, relative humidity $([0,100])$, temperature $(\mathcal{R})$, sine of wind direction $(\sin (\theta) \in[-1,1])$, cosine of wind direction $(\cos (\theta) \in[-1,1])$, and wind speed $\left(\mathcal{R}^{+}\right)$. The purpose of taking sine and cosine of the wind direction is to mitigate the discontinuity of angular values (for instance, $2 \pi$ and 0 are different values but representing the same direction), which impose difficulties for gradient-based parameter optimization techniques. The outputs are the predicted air pollutant levels of the future 24 hours.

It is a large field for users to explore the different designs of the NNs structure: for example, the deep long short-term memory (LSTM) network is commonly applied for multivariate timeseries regression. Furthermore, there are various optimization and regularization techniques one can apply. Since we focus on integrating the output of the NN into the belief updating framework, the analysis of design choices of NNs are beyond the scope of this article.

\section{EXAMPLES AND EXPERIMENTS}

In this section, we present numerical examples and experimental results. We divide the entire dataset (from 2006 to 2015) into two subsets: training data (2006 to 2012, 7 years) and testing data (2013 to 2015, 3 years). The mean function, covariance function, and the NN used in the models are learned based on the training set. The experiment are implemented in Python 3.6, and running on a computer with 16 processors $(2.3 \mathrm{GHz})$ and $32 \mathrm{~GB}$ memory. For the neural network part, we use the TensorFlow and Keras library.

\subsection{Examples}

First we present an illustrative example (7) of predicting the future 24-hours air pollutants, using the iterative belief updating with different data sizes. 

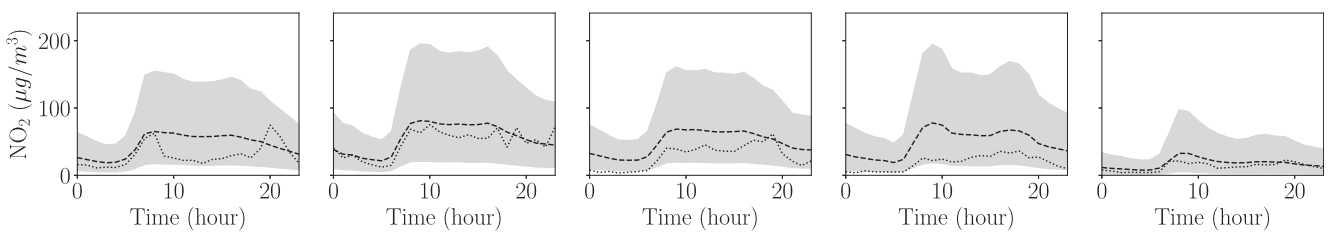

(a) The empirical prior
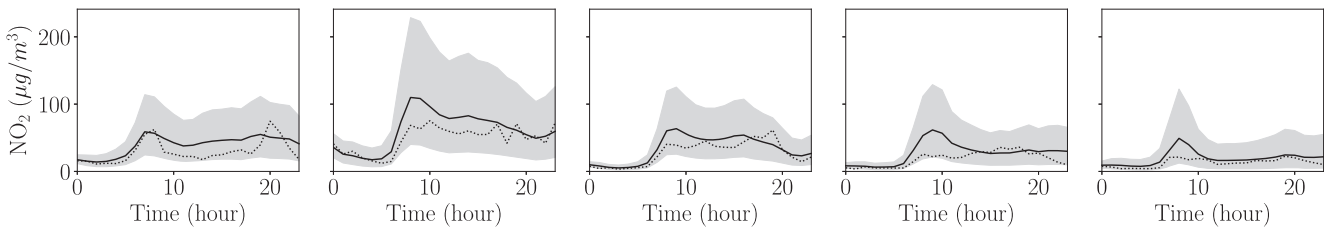

(b) The posterior based on the previous 1 week data
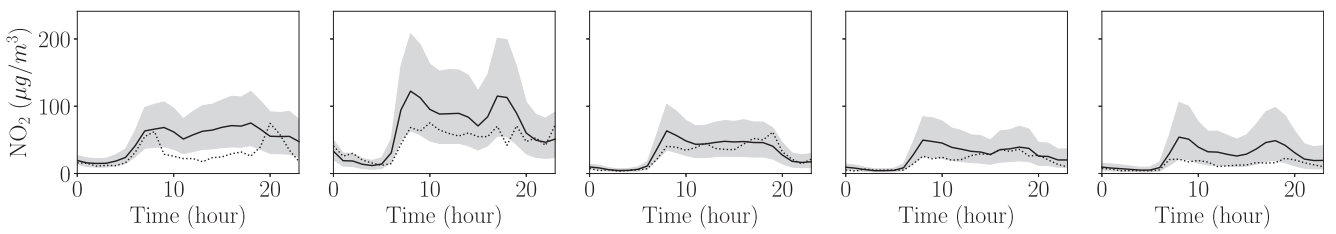

(c) The iteratively updated belief based on the previous 10 week data (1 week per iteration)
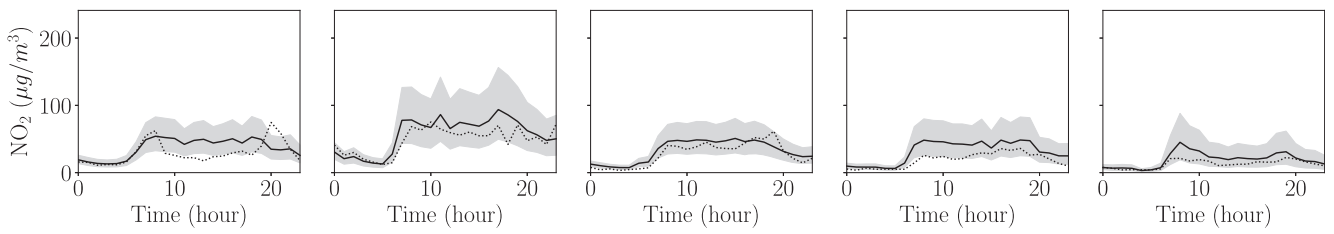

(d) The iteratively updated belief based on the previous 80 week data (8 week per iteration)

Fig. 7. The prior and updated beliefs of the predicted $\mathrm{NO}_{2}$ level for March 20, 2013 (Wednesday) at Stations 1-5 (from left to right column). The empirical prior is constructed based on the data before 2013. The updated beliefs are formed using data of various sizes and batch sizes. The solid lines are predictive means; the dot lines are true values; and the gray areas are the data between 95 and 5 percentiles.

In this example, we are interested in not only the accuracy of predictive values but also the validity of the predictions. Therefore, the results are represented using the probabilistic distributions, instead of only point values. Specifically, we focus on the following aspects of obtained updated belief distributions:

- The Level of Uncertainty. This aspect can be measured by the dispersion of a belief distribution. Specifically, in this example, we calculate the low (5) and high (95) percentiles, and using the distance between those low and high percentiles (90\% credible interval) to describe the level of uncertainty. A narrow credible interval indicates a low uncertain level of the updated belief distribution. 
Table 2. The Median of Absolute Errors of 24-hours $\mathrm{NO}_{2}$ Predictions Produced by the Scalable Belief Updating Method with Various Size of Data

\begin{tabular}{cccccc}
\hline & \multicolumn{5}{c}{ Median of Absolute Error $\left(\mu \mathrm{g} / \mathrm{m}^{3}\right)$} \\
\hline Data Size (weeks) & Station 1 & Station 2 & Station 3 & Station 4 & Station 5 \\
10 & 16.87 & 18.62 & 17.48 & 20.03 & 10.05 \\
40 & 13.33 & 16.87 & 13.53 & $\mathbf{1 5 . 6 8}$ & 7.07 \\
80 & 12.42 & 16.46 & $\mathbf{1 3 . 1 8}$ & 16.20 & 7.40 \\
160 & $\mathbf{1 2 . 2 0}$ & $\mathbf{1 5 . 9 4}$ & 13.77 & 16.15 & 7.34 \\
\hline
\end{tabular}

The prior is set to the empirical prior constructed from the historical $\mathrm{NO}_{2}$ measurements.

- The Accuracy of Prediction. The credible interval not only shall be small, but also covers the true value of the air pollution levels to be predicted. One can also provide the mean or median of the updated belief distribution, if the point predictions are required.

Taking Figure 7(a) as the starting point, we observe that the prior belief has very large credible interval, especially for air pollution levels during the daytime. And the mean values of the prior beliefs for Stations 3 and 4 are far from the true values. By combining one-week data with the empirical prior, we show the Bayesian posterior in Figure 7(b), which does not provide satisfying level of uncertainty, nor accurate predictions. Next, we apply the proposed iterative method. In each iteration, one-week historical data is utilized to update the belief distribution. The result is shown in Figure 7(c): the dispersion of the updated beliefs are reduced significantly, however, the accuracy is still not satisfying. To further improve the quality of the updated beliefs, we can increase the size of data processed in each iteration. In Figure 7(d), we utilize eight-week historical data per iteration. The obtained belief distributions have much smaller dispersion and higher accuracy.

\subsection{The Impact of Increasing Data and Using Meteorology Condition}

In this section, we present the experimental results by repeating the 24-hours forward prediction during years 2013-2015 for both $\mathrm{NO}_{2}$ and $\mathrm{PM}_{10}$ at five monitoring stations. The empirical mean and covariance functions are estimated using the data collected during 2006-2012. We use the predictive mean as the point prediction, and calculate the median absolute error as the metric of prediction accuracy.

Increasing the Size of Data. First, we apply the proposed scalable belief updating method with the empirical Gaussian prior specified by model 5. By increasing the total data size (in number of weeks), Table 2 shows that the MAE of predictions is reduced in general. For example, the predictions using 160 weeks (26,880 hourly measurements) of data for Stations 1, 2, and 4 achieve the lowest compare to the predictive result using less data. It is important to notice that processing such a large data is computationally prohibitive when using the classic GP model, and therefore infeasible to calculate in practice. By applying the proposed scalable method, however, one can iteratively process the large dataset and produce accurate predictions. However, we do observe the diminishing information gain of integrating the very large dataset. For Stations 3 and 5, the accuracies of predicting $\mathrm{NO}_{2}$ using 160 weeks of data are even lower than using 80 or 40 weeks of data.

Impact of the Meteorology Conditions. Next, we replace the empirical GP prior in Equation (5) with the output of the NN which approximates $p\left(\boldsymbol{x}_{*} \mid \boldsymbol{y}_{t}\right)$. The NN used in this experiments is designed to be a 10-layer fully connected network. We train the NN using the Adam optimizer [16] with the early stopping technique. 
Table 3. The Median of Absolute Errors of 24-hours $\mathrm{PM}_{10}$ Predictions Produced by the Scalable Belief Updating Method with Various Size of Data

\begin{tabular}{cccccc}
\hline & \multicolumn{5}{c}{ Median of Absolute Error $\left(\mu \mathrm{g} / \mathrm{m}^{3}\right)$} \\
\hline Data Size (weeks) & Station 1 & Station 2 & Station 3 & Station 4 & Station 5 \\
10 & 16.04 & 26.66 & 11.49 & 15.98 & 5.91 \\
40 & 10.28 & $\mathbf{1 2 . 3 2}$ & $\mathbf{1 0 . 1 6}$ & $\mathbf{1 0 . 6 5}$ & $\mathbf{5 . 6 0}$ \\
80 & $\mathbf{9 . 8 6}$ & 13.37 & 10.49 & 11.01 & 5.64 \\
160 & 10.53 & 17.66 & 11.22 & 11.86 & 6.38 \\
\hline
\end{tabular}

The prior is set to the empirical prior constructed from the historical $\mathrm{PM}_{10}$ measurements.

Table 4. The Median of Absolute Errors of 24-hours $\mathrm{NO}_{2}$ Predictions Produced by the Scalable Belief Updating Method with Various Size of Data

\begin{tabular}{cccccc}
\hline & \multicolumn{5}{c}{ Median of Absolute Error $\left(\mu \mathrm{g} / \mathrm{m}^{3}\right)$} \\
\hline Data Size (weeks) & Station 1 & Station 2 & Station 3 & Station 4 & Station 5 \\
10 & 15.27 & 17.08 & 15.21 & 18.54 & 9.68 \\
40 & 11.74 & 15.68 & 12.27 & $\mathbf{1 4 . 6 7}$ & $\mathbf{6 . 8 1}$ \\
80 & 11.07 & 15.61 & $\mathbf{1 2 . 1 9}$ & 14.98 & 7.58 \\
160 & $\mathbf{1 1 . 0 4}$ & $\mathbf{1 5 . 4 7}$ & 14.56 & 16.20 & 7.89 \\
\hline
\end{tabular}

The prior is set to the output of the $\mathrm{NN}$ which approximates the conditional distribution of $\mathrm{NO}_{2}$ given meteorology data.

Table 5. The Median of Absolute Errors of 24-hours $\mathrm{PM}_{10}$ Predictions Produced by the Scalable Belief Updating Method with Various Size of Data

\begin{tabular}{cccccc}
\hline & \multicolumn{5}{c}{ Median of Absolute Error $\left(\mu \mathrm{g} / \mathrm{m}^{3}\right)$} \\
\hline Data Size (weeks) & Station 1 & Station 2 & Station 3 & Station 4 & Station 5 \\
10 & 15.01 & 24.77 & 10.24 & 12.02 & 5.84 \\
40 & 10.28 & $\mathbf{1 2 . 3 2}$ & 10.20 & $\mathbf{9 . 3 2}$ & $\mathbf{5 . 6 5}$ \\
80 & $\mathbf{1 0 . 2 9}$ & 13.66 & $\mathbf{1 0 . 0 7}$ & 9.74 & 5.82 \\
160 & 10.38 & 15.19 & 10.53 & 10.47 & 6.55 \\
\hline
\end{tabular}

The prior is set to the output of the NN which approximates the conditional distribution of $\mathrm{PM}_{10}$ given meteorology data.

Comparing Table 2 with Table 4, we observe that the prediction accuracies for $\mathrm{NO}_{2}$ are considerably improved by integrating the information from the meteorology data. Recall the correlation analysis in Table 1, temperature and wind speed have high negative correlations with the concentration of $\mathrm{NO}_{2}$, which indicates the existence of rich information for $\mathrm{NO}_{2}$ in the meteorology data.

However, as Table 1 also shows, the correlation between meteorology conditions and the $\mathrm{PM}_{10}$ concentrations are much lower than the $\mathrm{NO}_{2}$ case. This analysis is reflected by the comparison of Tables 3 and 5: by integrating the meteorology data, the improvement of accuracies for $\mathrm{PM}_{10}$ is far less significant than the $\mathrm{NO}_{2}$ case.

A Comparison between the Run-Times of SGP and Recursive Update. We compare the run-times of predicting 24-hour air pollution using various size of historical data, using the exact likelihood, the sparse loss, and the recursive update. Using the exact likelihood, both the processing time and memory requirements increase drastically when the utilized data size grows from 8 to 56 weeks. In contrast, the runtime of the proposed recursive update method grows linearly and become nearly 


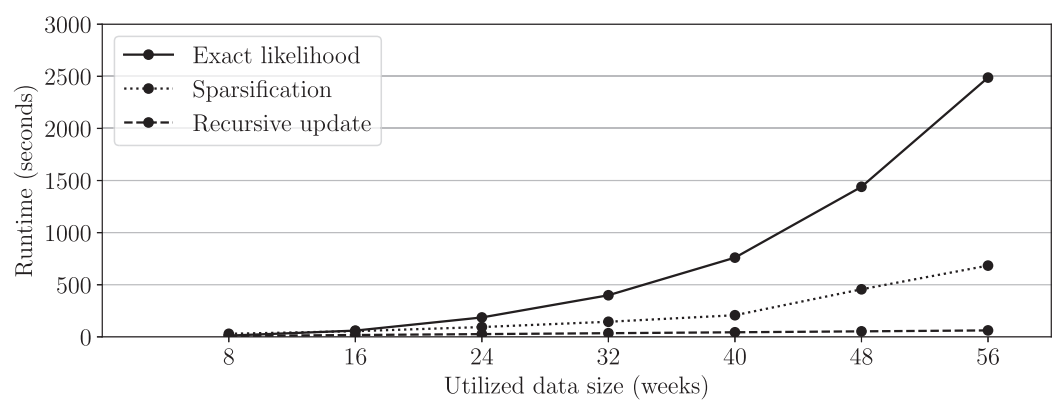

Fig. 8. A comparison of the the runtimes (per 24-hours prediction) provided by the exact likelihood, the sparsification, and the recursive update.
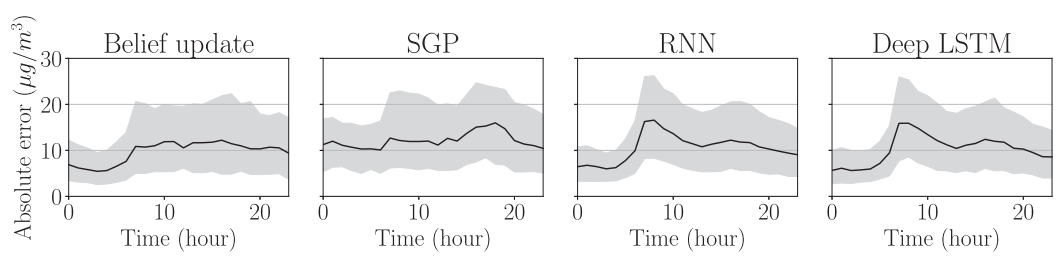

(a) $\mathrm{NO}_{2}$
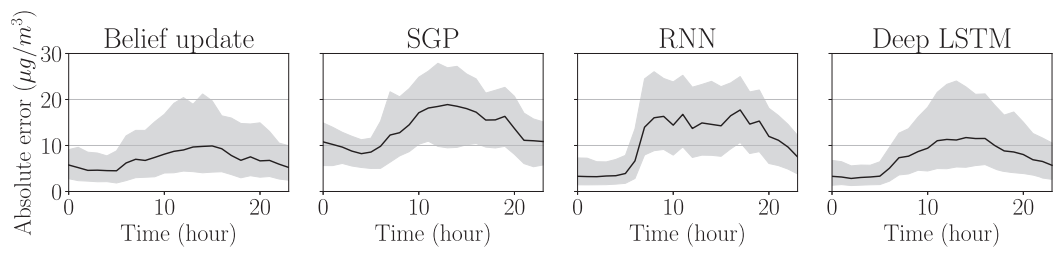

(b) $\mathrm{PM}_{10}$

Fig. 9. The 24-hour absolute prediction errors at Station 1, given by the scalable belief update, the SGP, the standard RNN, and the deep LSTM. The solid lines show the median of the absolute prediction errors for all test dates; the shadow areas show the 25 and 75 percentiles of the absolute errors.

50 times faster than the exact updates. The runtime of the sparse loss function is significantly lower than the exact likelihood, but much higher than the recursive update.

\subsection{Comparisons with Other Baseline Models}

Finally, we compare the performance of the proposed scalable belief update method with three baseline models: the sparse GP [3], the standard recurrent neural network [8, 9, 13], and the deep long short-term memory (LSTM) network [11, 18, 23].

The 24-Hour Prediction Errors. We start with comparing the distribution of the 24-hour absolute prediction errors. For illustrative purpose, we only show the prediction results for $\mathrm{NO}_{2}$ and $\mathrm{PM}_{10}$ at Station 1 in Figure 9, where the solid lines are the median of the absolute errors and the gray areas represent the 25 to 75 percentiles.

For $\mathrm{NO}_{2}$ predictions, the proposed belief update method achieve lowest median errors and 75 percentiles among the compared methods. The morning rush hours (around 8 to 10) has the largest variance, which implies large uncertainty and high difficulty for making accurate 

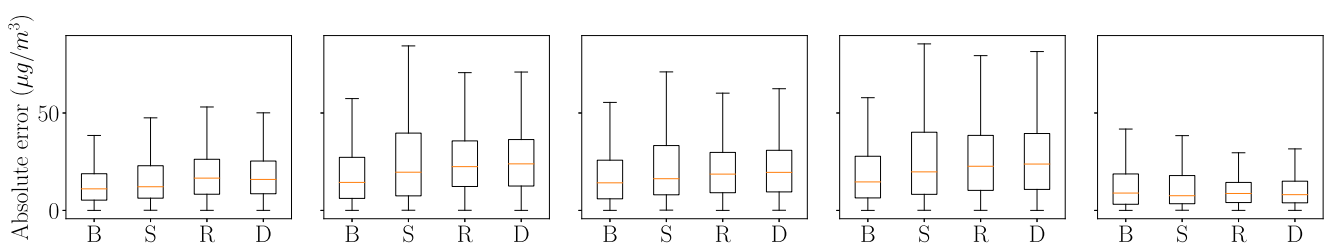

(a) $\mathrm{NO}_{2}$
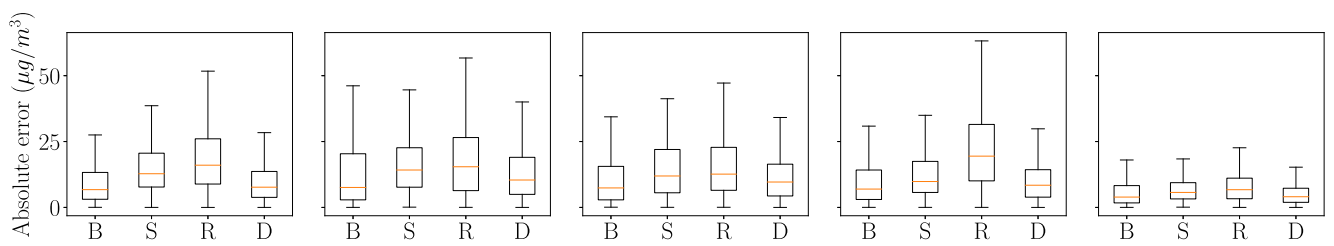

(b) $\mathrm{PM}_{10}$

Fig. 10. The distributions of rush hours prediction errors at Station 1-5 (from left to right). Four models are compared: the proposed Belief updating (B), the SGP (S), the standard RNN network (R), and the deep LSTM network (D). The whiskers show the 5 and the 95 percentiles.

predictions. The scalable belief update method does not show peaks of its median absolute error at the morning rush hours. This result indicate that the proposed model is highly flexible and capable of capturing the high-frequency periodic patterns in the data (the 12-hour and the 8-hour patterns in Figure 4(a)). On the other hand, both the standard RNN and the deep LSTM models show notable peaks of prediction errors around the morning rush hours. Furthermore, for the $\mathrm{NO}_{2}$ prediction, the deep LSTM model dose not show significant improvement of accuracy, when it is compared to the standard RNN model.

For $\mathrm{PM}_{10}$ predictions, the proposed belief update method and the deep LSTM model have similar performances, which have significantly lower median and percentiles than the SGP and the standard RNN models. The $\mathrm{PM}_{10}$ has a different dispersion mechanism than the $\mathrm{NO}_{2}$, and does not have obvious peaks around the morning and the afternoon rush hours. This is also reflected in the PSD plot of $\mathrm{PM}_{10}$ in Figure 4(b). In general, the prediction's errors peak around the noon time. And the proposed belief update method shows the lowest peak of absolute prediction error among all the methods.

The Distribution of Rush Hours Prediction Errors. To exam the models' performances around the test points with maximum uncertainties, we illustrate the distributions of rush hours prediction errors in Figure 10.

For $\mathrm{NO}_{2}$ predictions, the proposed scalable belief update method achieve the best performances except for Station 5. However, since Station 5 is a background air quality monitoring station, its measurements are in general low and less interesting for urban citizens. For Stations 1-4 which are deployed in the city center, the proposed belief updating method is able to produce predictions with the lowest median and the smallest variance of absolute errors.

For $\mathrm{PM}_{10}$ predictions, the proposed scalable belief update method and the deep LSTM network achieve comparable results for all stations. We notice that the standard RNN network has the highest median of absolute errors, especially for Station 4.

\section{CONCLUSIONS AND FUTURE WORK}

In this article, we presented a scalable belief updating framework for predicting urban air quality $\left(\mathrm{NO}_{2}\right.$ and $\left.\mathrm{PM}_{10}\right)$. We started with a descriptive statistical analysis of the air pollution data. The time 
and frequency domain analysis revealed interesting periodic dynamic patterns in the air pollution data. The correlations between the air pollution and the meteorological conditions were further explored.

Based on those information, we proposed a scalable belief updating framework which accommodates the popular GP model for multivariate time series forecast. The proposed framework uses the composite likelihood as the loss function, which enables iterative updates of the posterior distributions. Compared to the classical GP model and sparse GP model, the proposed method scales better and avoids heuristic search for obtaining sparse representations of the whole dataset.

Furthermore, we demonstrated how to integrate information from heterogeneous data (e.g., meteorological data in this study), using informative prior beliefs produced by deep neural networks. Given the significant correlation between $\mathrm{NO}_{2}$ and the meteorological conditions, the experimental results showed notable improvement in the prediction accuracy for $\mathrm{NO}_{2}$.

For future works, it would be interesting to investigate the exact information loss by using the composite likelihood in the scalable belief updating framework. This analysis could help to understand the limit of approximating the exact likelihood with the composite one. Another interesting direction is to design and apply more sophisticated NN structures which are capable of representing the conditional distribution of air pollutants given the meteorological conditions.

\section{ACKNOWLEDGMENTS}

We thank the editor and anonymous reviewers for their constructive comments.

\section{REFERENCES}

[1] U. S. Environmental Protection Agency. 1999. Nitrogen Oxides (NOx), Why and How They Are Controlled.

[2] V. Athira, P. Geetha, R. Vinayakumar, and K. P. Soman. 2018. Deepairnet: Applying recurrent networks for air quality prediction. Procedia Computer Science 132 (2018), 1394-1403.

[3] Matthias Bauer, Mark van der Wilk, and Carl Edward Rasmussen. 2016. Understanding probabilistic sparse Gaussian process approximations. In Advances in Neural Information Processing Systems. 1533-1541.

[4] Christopher M. Bishop. 2006. Pattern Recognition and Machine Learning. Springer.

[5] Pier Giovanni Bissiri, Chris C. Holmes, and Stephen G. Walker. 2016. A general framework for updating belief distributions. Journal of the Royal Statistical Society: Series B (Statistical Methodology) 78, 5 (2016), 1103-1130.

[6] George E. P. Box. 1979. Robustness in the strategy of scientific model building. In Robustness in Statistics. Elsevier, 201-236.

[7] George E. P. Box and David R. Cox. 1964. An analysis of transformations. Fournal of the Royal Statistical Society: Series B (Methodological) 26, 2 (1964), 211-243.

[8] Tien-Cuong Bui, Van-Duc Le, and Sang-Kyun Cha. 2018. A deep learning approach for forecasting air pollution in south korea using LSTM. Arxiv Preprint Arxiv:1804.07891 (2018).

[9] Jerome T. Connor, R. Douglas Martin, and Les E. Atlas. 1994. Recurrent neural networks and robust time series prediction. IEEE Transactions on Neural Networks 5, 2 (1994), 240-254.

[10] Paulo S. R. Diniz, Eduardo A. B, Da Silva, and Sergio L. Netto. 2010. Digital Signal Processing: System Analysis and Design. Cambridge University Press.

[11] Yanjie Duan, Yisheng Lv, and Fei-Yue Wang. 2016. Travel time prediction with LSTM neural network. In 2016 IEEE 19th International Conference on Intelligent Transportation Systems (ITSC). IEEE, 1053-1058.

[12] Dongbing Gu and Huosheng Hu. 2012. Spatial gaussian process regression with mobile sensor networks. IEEE Transactions on Neural Networks and Learning Systems 23, 8 (2012), 1279-1290.

[13] Min Han, Jianhui Xi, Shiguo Xu, and Fu-Liang Yin. 2004. Prediction of chaotic time series based on the recurrent predictor neural network. IEEE Transactions on Signal Processing 52, 12 (2004), 3409-3416.

[14] Ching-Hui Huang, Heng-Cheng Lin, Chen-Dao Tsai, Hung-Kai Huang, Ie-Bin Lian, and Chia-Chu Chang. 2017. The interaction effects of meteorological factors and air pollution on the development of acute coronary syndrome. Scientific Reports 7 (2017), 44004.

[15] Rishee K. Jain, Jose M. F. Moura, and Constantine E. Kontokosta. 2014. Big data+ big cities: Graph signals of urban air pollution [exploratory sp]. IEEE Signal Processing Magazine 31, 5 (2014), 130-136.

[16] Diederik P. Kingma and Jimmy Ba. 2014. Adam: A method for stochastic optimization. Arxiv Preprint Arxiv:1412.6980 (2014). 
[17] Andreas Krause, Ajit Singh, and Carlos Guestrin. 2008. Near-optimal sensor placements in gaussian processes: Theory, efficient algorithms and empirical studies. Journal of Machine Learning Research 9, Feb. (2008), 235-284.

[18] Yuxuan Liang, Songyu Ke, Junbo Zhang, Xiuwen Yi, and Yu Zheng. 2018. Geoman: Multi-level attention networks for geo-sensory time series prediction. In Proceedings of IFCAI. 3428-3434.

[19] Haitao Liu, Yew-Soon Ong, Xiaobo Shen, and Jianfei Cai. 2018. When Gaussian process meets big data: A review of scalable GPs. Arxiv Preprint Arxiv:1807.01065 (2018).

[20] M. Lototzis, G. K. Papadopoulos, F. Droulia, A. Tseliou, and I. X. Tsiros. 2018. A note on the correlation between circular and linear variables with an application to wind direction and air temperature data in a mediterranean climate. Meteorology and Atmospheric Physics 130, 2 (2018), 259-264.

[21] World Health Organization. 2015. Economic cost of the health impact of air pollution in Europe: Clean air, health and wealth.

[22] C. Arden Pope III and Douglas W. Dockery. 1992. Acute health effects of PM10 pollution on symptomatic and asymptomatic children. American Review of Respiratory Disease 145, 5 (1992), 1123-1128.

[23] Zhongang Qi, Tianchun Wang, Guojie Song, Weisong Hu, Xi Li, and Zhongfei Zhang. 2018. Deep air learning: Interpolation, prediction, and feature analysis of fine-grained air quality. IEEE Transactions on Knowledge and Data Engineering 30, 12 (2018), 2285-2297.

[24] Joaquin Quiñonero-Candela and Carl Edward Rasmussen. 2005. A unifying view of sparse approximate Gaussian process regression. fournal of Machine Learning Research 6, Dec (2005), 1939-1959.

[25] Carl Edward Rasmussen and Christopher Williams. 2006. Gaussian Processes for Machine Learning. The MIT Press.

[26] Matthias Seeger, Christopher Williams, and Neil Lawrence. 2003. Fast Forward Selection to Speed up Sparse Gaussian Process Regression. Technical Report.

[27] Khaled Bashir Shaban, Abdullah Kadri, and Eman Rezk. 2016. Urban air pollution monitoring system with forecasting models. IEEE Sensors fournal 16, 8 (2016), 2598-2606.

[28] Robert H. Shumway and David Stoffer. 2017. Time Series Analysis and Its Applications: with R Examples. Springer.

[29] Edward Snelson and Zoubin Ghahramani. 2006. Sparse Gaussian processes using pseudo-inputs. In Advances in Neural Information Processing Systems. 1257-1264.

[30] In-Kwon Yeo and Richard A. Johnson. 2000. A new family of power transformations to improve normality or symmetry. Biometrika 87, 4 (2000), 954-959.

[31] Xiuwen Yi, Junbo Zhang, Zhaoyuan Wang, Tianrui Li, and Yu Zheng. 2018. Deep distributed fusion network for air quality prediction. In Proceedings of the 24th ACM SIGKDD International Conference on Knowledge Discovery \& Data Mining. 965-973.

[32] Yu Zheng, Licia Capra, Ouri Wolfson, and Hai Yang. 2014. Urban computing: Concepts, methodologies, and applications. ACM Transactions on Intelligent Systems and Technology (TIST) 5, 3 (2014), 38.

[33] Yu Zheng, Furui Liu, and Hsun-Ping Hsieh. 2013. U-air: When urban air quality inference meets big data. In Proceedings of the 19th ACM SIGKDD International Conference on Knowledge Discovery and Data Mining. ACM, 1436-1444.

[34] Yu Zheng, Xiuwen Yi, Ming Li, Ruiyuan Li, Zhangqing Shan, Eric Chang, and Tianrui Li. 2015. Forecasting finegrained air quality based on big data. In Proceedings of the 21th ACM SIGKDD International Conference on Knowledge Discovery and Data Mining. ACM, 2267-2276.

Received June 2019; revised March 2020; accepted May 2020 